\title{
Strategies for prevention of prolonged intensive care unit stay following cardiac surgery by identifying the determinants
}

\author{
Philemon Gukop ${ }^{1 *}$, Oswaldo Valencia', Mazin Sarsam¹, Vankatachallam Chandrasekaran', Brendan Madden² \\ From World Society of Cardiothoracic Surgeons 25th Anniversary Congress, Edinburgh \\ Edinburgh, UK. 19-22 September 2015
}

\section{Background/Introduction}

Cardiac surgery service is dependent on the availability of cardiac intensive care facility. some patients are eligible for fast-track protocol. We investigate the factors determining prolonged intensive care stay following cardiac surgery, with the view to developing a model that predicts prolonged stay.

\section{Aims/Objectives}

Develop a scoring model that predicts prolonged intensive care stay following cardiac surgery

\section{Method}

Retrospective data analysis on 1592 consecutive patients admitted to intensive care following cardiac surgery (2011-2014). Dichotomous and categorical data were compared using Chi-square or Fisher's Exact tests. P-value of $<0.05$ was significant. Univariate and Multivariate Regression identified predictors of prolonged intensive care stay.

A score model for prolonged intensive care stay was developed as a logistic probability unit $(\mathrm{z}=\operatorname{logit}(\mathrm{p})=\log$ e (p/1-p); The area under the receiver curve (AUC) generated. The best cut-off point of the scoring model was identified, the likelihood ratio of a positive test result calculated.

\section{Results}

Logistic regression showed predictors of prolonged intensive care unit stay as ; NYHA class 3-4 (OR,1.5; $\mathrm{p}=0.0029)$, FEV1 (OR, 0.76; $\mathrm{p}=0.0026)$, emergency operation $(\mathrm{OR}, 8.75 ; \mathrm{p}=0.0022)$, age $(\mathrm{OR}, 1.02$; $\mathrm{p}=0.00007), \mathrm{LVEF}<50 \%(\mathrm{OR}, 2.21 ; \mathrm{p}=0.00001)$, creatinine $(\mathrm{OR}, 1.01 ; \mathrm{p}=0.000001)$, bypass time $(\mathrm{OR}, 1.01$; $\mathrm{p}=0.000001)$.

Intensive care unit stay score was determined by logistic probability $(\mathrm{AUC}=0.76,95 \% \mathrm{CI}, 0.73 ; 0.78, \mathrm{p}=$ 0.00001 ) suggesting that a cut-off score of 35 predicts prolonged intensive care stay with a sensitivity of 0.66 , specificity of 0.72 and accuracy of 0.70 . The likelihood ratio of a positive test was 2.34 .

\section{Discussion/Conclusion}

Preoperative optimisation of the predictors of prolong intensive care stay, could reduce length of stay following cardiac-surgery.

\section{Authors' details}

'Department of Cardiothoracic Surgery, St George's University Hospital NHS Foundation Trust, London, SW17 0QT, UK. ²Department of Cardiorespiratory Medicine and Intensive Care, St George's University Hospital NHS Foundation Trust, London, SW17 OQT, UK.

Published: 16 December 2015

doi:10.1186/1749-8090-10-S1-A165

Cite this article as: Gukop et al:: Strategies for prevention of prolonged intensive care unit stay following cardiac surgery by identifying the determinants. Journal of Cardiothoracic Surgery 2015 10(Suppl 1):A165. 